Pacific Journal of Mathematics

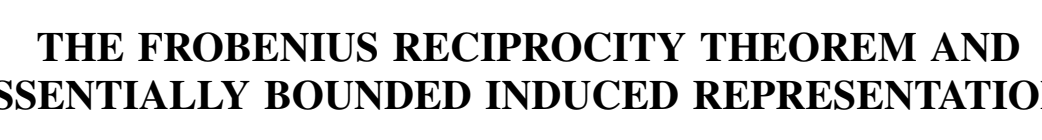




\title{
THE FROBENIUS RECIPROCITY THEOREM AND ESSENTIALLY BOUNDED INDUCED REPRESENTATIONS
}

\author{
William L. Armacost
}

Let $G$ be a locally compact group with continuous unitary representations $R_{i}$ acting on the Hilbert spaces $H\left(R_{\imath}\right), i=1,2$. Suppose that $R_{2}$ is irreducible. A closed subspace of $H\left(R_{1}\right)$, called the null space coming from $R_{1}$ at $R_{2}$ and denoted $F\left(R_{1} ; R_{2}\right)$, is defined. Write $\mathscr{H}^{c}$ for the conjugate space of the Hilbert space $\mathscr{K}$. The following theorem is proved.

Theorem 1. Let $G$ be a compact group with closed subgroup $H$. Let $M, L$ be irreducible unitary representations of $G, H$, respectively. Let $U^{L}$ be the induced representation of $L$, and let $M_{H}$ be the restriction of $M$ to $H$. Then the following are equivalent:

(a) The classical Frobenius Reciprocity Theorem.

(b) $H(M) \otimes F\left(M_{\Pi} ; L\right)^{c} \cong F\left(U^{L} ; M\right) \otimes H(L)^{c}$.

When $G$ is not compact, both (a) and (b) may fail. A non-Hilbert Banach space induced representation $\left(W^{L}\right)$ is defined. Let $G$ be a locally compact group with closed subgroup $H$. Let $M, L$ be irreducible unitary representations of $G, H$, respectively, where $H(L)$ is separable. Spaces $F_{0}\left(W^{L}\right.$; $M) \otimes * H(L)^{c}$ (shown to equal $F\left(U^{L} ; M\right) \otimes H(L)^{c}$ when $G$ is compact) and $Q F\left(M_{H} ; L\right)$ shown to equal $F\left(M_{H} ; L\right)$ when $L$ is compact) are defined. The following generalization of (b) is shown.

Theorem 2. Let $G, H, L$ be as above. Then

$$
H(M) \otimes Q F\left(M_{H} ; L\right)^{c} \cong F_{0}\left(W^{L} ; M\right) \otimes^{*} H(L)^{c} .
$$

As these results indicate, the purpose of this paper is to establish yet another generalization of the Frobenius Reciprocity Theorem (FRT). The genesis of our approach is two-fold. The idea of employing the null space was suggested by Blattner's study [1] of the Glimm projection-valued measure. The notion of defining a nonHilbert Banach space induced representation seemed natural in light of the work of Moore [8] and Kleppner [7].

We recall the following basic concepts. Suppose $G$ is a locally compact group (we denote its neutral element by $e$ ). Endowing $G$ with right invariant Haar measure, one uses the familiar notions of convolution and * to define two Banach algebras, $L_{1}(G)$ (=the $L_{1}$-group algebra of $G$ with norm denoted by $\|\cdot\|_{1}$ ) and $C^{*}(G)$ 
(= the $C^{*}$-group algebra of $G$ with norm denoted by $\|\cdot\|_{*}$ ). Let $R$ be a unitary (always assumed continuous) representation of $G$, and denote always by $H(R)$ the Hilbert space on which $R$ acts. In terms of such a representation $R$ of $G$, we define nondegenerate continuous *-representations (denoted by $R$ again) of $L_{1}(G)$ and $C^{*}(G)$ in the usual way. The representation theories of $L_{1}(G)$ and $C^{*}(G)$ mirror that of $G$. The reader is referred to [1] for a precise statement of these facts.

1. The null space. Suppose $\mathscr{A}$ is a $C^{*}$-algebra. The Jacobson structure space of $\mathscr{A}$, written $\mathscr{A}^{*}$, is defined to be the space of kernels of irreducible nondegenerate ${ }^{*}$-representations of $\mathscr{A}$ endowed with the hull-kernel topology. If $R$ is a *-representation acting on the Hilbert space $H(R)$, Glimm [5] has shown that there exists a unique projection-valued measure $E(R ; \cdot)$ on the Borel field generated by the topology of $\mathscr{A}^{*}$ such that if $C$ is a closed subset of $\mathscr{A}^{*}$, $E(R ; C)$ is the projection on the manifold

$$
F(R ; C)=\{v \in H(R): R(a) v=0 \text { for all } a \in \bigcap C\} .
$$

$E(R ; \cdot)$ takes its values in the center of the von-Neumann algebra generated by $R(\mathscr{A})$.

Let $G$ be a locally compact group with unitary representations $R_{1}, R_{2}$; suppose $R_{2}$ is irreducible. Regarding $R_{1}$ and $R_{2}$ as representations of $C^{*}(G)$ as well as of $G$, we consider the space $F\left(R_{1} ; \mathrm{Cl}\left\{\operatorname{Ker} R_{2}\right\}\right)$, where $\mathrm{Cl}\left\{\operatorname{Ker} R_{2}\right\}$ is the closure in the structure space topology of the point-set containing the kernel of the representation $R_{2}$, $\operatorname{Ker} R_{2}$. Because $\operatorname{Ker} R_{2} \in \mathrm{Cl}\left\{\operatorname{Ker} R_{2}\right\}$, we have

$$
F\left(R_{1} ; \mathrm{Cl}\left\{\operatorname{Ker} R_{2}\right\}\right)=\left\{v \in H\left(R_{1}\right): R_{1}(f) v=0 \text { for all } f \in \operatorname{Ker} R_{2}\right\} .
$$

The projection $E\left(R_{1} ; \mathrm{Cl}\left\{\operatorname{Ker} R_{2}\right\}\right)$ will be called the null projection coming from $R_{1}$ at $R_{2}$, and will be denoted by $E\left(R_{1} ; R_{2}\right)$. The space $F\left(R_{1} ; \mathrm{Cl}\left\{\operatorname{Ker} R_{2}\right\}\right)$ will be called the null space coming from $R_{1}$ at $R_{2}$ and will be denoted by $F\left(R_{1} ; R_{2}\right)$.

LeMma 1. Let $G$ be a compact group. Let $R_{1}$ be a unitary representation of $G$ which contains a unitary irreducible representation $R_{2}$ of $G$ exactly $n$ times. Then $F\left(R_{1} ; R_{2}\right) \cong n H\left(R_{2}\right)$.

Proof. For a compact group, the fact that $R_{1}$ contains $R_{2}$ exactly $n$ times means that $R_{1}$ is unitarily equivalent to a discrete direct sum of representations as follows: $R_{1} \cong n R_{2} \oplus\left(\oplus_{\alpha} R_{\alpha}\right)$, where each $R_{\alpha}$ is an irreducible unitary representation of $G$ disjoint from $R_{2}$. For $v \in H\left(R_{1}\right)$, write 


$$
v=\bigoplus_{i=1}^{n} v_{i} \oplus\left(\bigoplus_{\alpha} v_{\alpha}\right), v_{i} \in H\left(R_{2}\right), v_{\alpha} \in H\left(R_{\alpha}\right),
$$

whence

$$
F\left(R_{1} ; R_{2}\right)=\bigoplus_{i=1}^{n}\left\{v_{i} \in H\left(R_{2}\right): R_{2}(f) v_{i}=0 \text { for all } f \in \operatorname{Ker} R_{2}\right\}
$$

$\bigoplus \bigoplus_{\alpha}\left\{v_{a} \in H\left(R_{\alpha}\right): R_{\alpha}(f) v=0\right.$ for all $\left.f \in \operatorname{Ker} R_{2}\right\}$. Since the first $n$ summands clearly sum to $n H\left(R_{2}\right)$, we need only show that for each $\alpha$, the spaces $F\left(R_{\alpha} ; R_{2}\right)$ consist of zero only. Because $E\left(R_{\alpha} ; R_{2}\right)$ commutes with $R_{\alpha}(f)$ for all $f \in C^{*}(G)$, Schur's lemma implies that $E\left(R_{\alpha} ; R_{2}\right)$ is either the zero operator or the identity operator $I_{\alpha}$ on $H\left(R_{\alpha}\right)$. If $E\left(R_{\alpha} ; R_{2}\right)=I_{\alpha}$, then $H\left(R_{\alpha}\right)=F\left(R_{\alpha} ; R_{2}\right)$, so that $\operatorname{Ker} R_{\alpha} \supseteqq$ $\operatorname{Ker} R_{2}$. This implies (see Fell [4], p. 378) that $\operatorname{Ker} R_{\alpha} \cong \operatorname{Ker} R_{2}$, which in turn implies (see Glimm [6], p. 583) that $R_{2} \cong R_{\alpha}$. This contradiction means $F\left(R_{\alpha} ; R_{2}\right)=\{0\}$, as we wanted.

Let $G$ be a locally compact group with unitary representation $R_{1}$ and irreducible unitary representation $R_{2}$. Since we will deal with the $L_{1}$-group algebra of $G$, let $\mathrm{Ker}_{1}$ denote the kernel of a *-representation of $L_{1}(G)$. The closed subspace $F_{1}\left(R_{1} ; R_{2}\right)$ of $H\left(R_{1}\right)$ defined by $F_{1}\left(R_{1} ; R_{2}\right)=\left\{v \in H\left(R_{1}\right): R_{1}(f) v=0\right.$ for all $\left.f \in \operatorname{Ker}_{1} R_{2}\right\}$ will be called the $L_{1}$-null space coming from $R_{1}$ at $R_{2}$. The projection $E\left(R_{1} ; R_{2}\right)$ on $F_{1}\left(R_{1} ; R_{2}\right)$ commutes with $R_{1}(f)$ for all $f \in L_{1}(G)$. The results of Fell and Glimm cited in the proof of Lemma 1 can be shown to remain valid when $C^{*}(G)$ is replaced by $L_{1}(G)$ if $R_{2}$ is of finite degree. If $G$ is a compact group, the fact that all of its irreducible representations are of finite degree will therefore mean that Lemma 1 remains true when $F\left(R_{1} ; R_{2}\right)$ is replaced by $F_{1}\left(R_{1} ; R_{2}\right)$.

Suppose now that $G$ is a compact group with closed subgroup $H$. Let $L$ be an irreducible unitary representation of $H$. Let $H\left(U^{L}\right)$ be the space of all Haar-measurable functions $f$ on $G$ with values in $H(L)$ such that (i) $f(x \gamma)=L(x) f(\gamma)$ for all $x \in H, \gamma \in G$, and (ii) $\int_{G}\|f(\gamma)\|^{2} d \gamma<\infty$. As usual, let $U^{L}$, the induced representation of $L$, be the representation of $G$ acting on $H\left(U^{L}\right)$, where $\left(U^{L}(\gamma) f\right)\left(\gamma^{\prime}\right)=$ $f\left(\gamma^{\prime} \gamma\right)$ for all $f \in H\left(U^{L}\right), \gamma, \gamma^{\prime} \in G$. Suppose $M$ is an irreducible unitary representation of $G$; let $M_{H}$ be the restriction of $M$ to $H$. Suppose $U^{L}$ contains $M$ exactly $n$ times. By Lemma 1 and the FRT, we have $F\left(U^{L} ; M\right) \cong n H(M)$ and $F\left(M_{H} ; L\right) \cong n H(L)$. Using a trivial dimension argument, we have

* $H(M) \otimes F\left(M_{H} ; L\right) \cong F\left(U^{L} ; M\right) \otimes H(L)$, where " $\otimes$ " denotes the algebraic tensor product. Since $H(L)$ and $H(M)$ are finite-dimensional, it is clear that ${ }^{*}$ implies the FRT. Letting $\mathscr{C}^{c}$ be the conjugate space of a Hilbert space $\mathscr{C}$ (so that $\operatorname{dim} \mathscr{H}=\operatorname{dim} \mathscr{H}^{c}$ ), we get

THEOREM 1. Let $G$ be a compact group with closed subgroup $H$. Let $M, L$ be irreducible unitary representations of $G, H$, re- 
spectively. Then the following statements are equivalent:

(a) The Frobenius Reciprocity Theorem.

(b) $H(M) \otimes F\left(M_{H} ; L\right)^{c} \cong F\left(U^{L} ; M\right) \otimes H(L)^{c}$.

(c) $H(M) \otimes F_{1}\left(M_{H} ; L\right)^{c} \cong F_{1}\left(U^{L} ; M\right) \otimes H(L)^{c}$.

2. Essentially bounded induced representations. It is quite easy to see that statements (b) and (c) of Theorem 1 are false if we abandon the assumption that $G$ is compact. Indeed, let $G$ be the group of real numbers under addition and let $H=\{0\}$; let $M$ be any character of $G$ and let $L$ be the irreducible identity representation of $H$. Then $U^{L}$ is the right regular representation of $G$ acting on $L_{2}(G)$ and $F\left(U^{L} ; M\right)$ is the trivial subspace of $L_{2}(G)$ having dimension zero, whereas $F\left(M_{H} ; L\right)$ has dimension one. However, if one considers the right regular representation of $G$ acting on $L_{\infty}(G)$ instead of $L_{2}(G)$ one can define in the natural way a space " $F\left(U^{L} ; M\right)$ " having dimension one. We therefore define a new type of induced representation; we argue in Proposition 1 below that this definition is reasonable by re-examining the case when $G$ is compact.

DeFinition 1. Let $G$ be a locally compact group with closed subgroup $H$. Let $L$ be a unitary representation of $H$ and let $L_{\infty}(G ; H(L))$ be the Banach space of all Haar-measurable essentially bounded functions from $G$ to $H(L)$ (for $f$ in $L_{\infty}\left(G ; H(L)\right.$ ), let $\|f\|_{\infty}=$ ess sup $\|f(\gamma)\|)$. Let $B\left(W^{L}\right)$ be the Banach space consisting of all $g$ in $L_{\infty}(G ; H(L))$ such that $g(x \gamma)=L(x) g(\gamma)$ for all $x \in H, \gamma \in G$. Define a representation $W^{L}$ of $G$ on $B\left(W^{L}\right)$ by the rule $\left(W^{L}(\gamma) g\right)\left(\gamma^{\prime}\right)=g\left(\gamma^{\prime} \gamma\right)$ for all $g \in B\left(W^{L}\right), \gamma, \gamma^{\prime} \in G ; W^{L}$ is called the $L_{\infty}$-induced representation of $L$ acting on $B\left(W^{L}\right)$.

It is clear that $B\left(W^{L}\right)$ is invariant under $W^{L}$, and that $W^{L}$ maps $G$ into the isometries on $B\left(W^{L}\right)$. It should be noted that $W^{L}$ is not in general a strongly continuous representation of $G$. We can define $W^{L}$ as a representation of $L_{1}(G)$ by the rule that for $f \in L_{1}(G), W^{L}(f)$ is the operator on $B\left(W^{L}\right)$ sending $g \in B\left(W^{L}\right)$ to $W^{L}(f) g \in B\left(W^{L}\right)$, where

$$
\begin{aligned}
\left(W^{L}(f) g\right)(a) & =\int_{G} f(\gamma)\left(W^{L}\left(\gamma^{-1}\right) g\right)(a) d \gamma \\
& =\int_{G} f(\gamma) g\left(a \gamma^{-1}\right) d \gamma,
\end{aligned}
$$

for all $a \in G$. Because $\left\|W^{L}(f) g\right\|_{\infty} \leqq\|f\|_{1}\|g\|_{\infty}$, the representation $f \rightarrow W^{L}(f)$ is a strongly continuous representation of $L_{1}(G)$. Note that since in general $\|f\|_{*} \leqq\|f\|_{1}$, it does not seem to be the case that one can similarly define a strongly continuous representation of $C^{*}(G)$. It is for this reason that we will have to concern ourselves 
with $L_{1}(G)$ at the expense of $C^{*}(G)$.

As $\mathrm{L}_{\infty}(G ; H(L))$ can be regarded as the dual of $L_{1}(G ; H(L)$ ) (defined in the natural way) when $H(L)$ is separable, $W^{L}$ can be shown to be a weak-* continuous representation of $G$ when in fact $H(L)$ is separable.

3. The case $G$ is compact. Let $G$ be any locally compact group with closed subgroup $H$. Let $M, L$ be irreducible unitary representations of $G, H$, respectively. Let us define a space $F_{1}\left(W^{L} ; M\right)$ in the natural way:

$$
F_{1}\left(W^{L} ; M\right)=\left\{g \in B\left(W^{L}\right): W^{L}(f) g=0 \text { for all } f \in \operatorname{Ker} M\right\} .
$$

When $G$ is compact, we have the following result.

Proposition 1. Let $G, H, M$ and $L$ be as in Lemma 1. Then $F_{1}\left(U^{L} ; M\right)=F_{1}\left(W^{L} ; M\right)$.

Proof. Since $H(L)$ is finite-dimensional, we can write (relative to some basis of $H(L))$ every $g \in H\left(U^{L}\right)$ as a "column-vector valued" function $g=\left(g_{k}\right)$, where $g_{k} \in L_{2}(G), \quad k=1, \cdots, r=\operatorname{dim} H(L)$. It plainly suffices to show that $F_{1}\left(U^{L} ; M\right) \subseteq F_{1}\left(W^{L} ; M\right)$, so we will be done if we establish that if $g=\left(g_{k}\right) \in F_{1}^{\prime}\left(U^{L} ; M\right)$ then each $g_{k}$ is in fact continuous (and hence in $L_{\infty}(G)$ ).

We have $F_{1}\left(U^{L} ; M\right)=\left\{g \in H\left(U^{L}\right): U^{L}(f) g=0\right.$ for all $\left.f \in \operatorname{Ker}_{1} M\right\}$. Let $m=\operatorname{dim} H(M)$. There exist $m^{2}$ continuous functions $h_{i j}$ on $G$ of the form $h_{i j}(\gamma)=\left(M\left(\gamma^{-1}\right) u_{i}, v_{j}\right)\left(u_{i}, v_{j} \in H(M), i, j=1, \cdots, m\right)$ such that $\operatorname{Ker}_{1} M=\left\{f \in L_{1}(G): \int_{G} f(\gamma) h_{i j}(\gamma) d \gamma=0, i, j=1, \cdots, m\right\}$. Clearly, if $g=\left(g_{k}\right) \in H\left(U^{L}\right)$ and if each $g_{k}$ is a linear combination of the continuous functions $h_{i j}^{\prime}$ (where $h_{i j}^{\prime}(\gamma)=h_{i j}\left(\gamma^{-1}\right)$ ), then $U^{L}(f)=0$ for all $f \in \operatorname{Ker}_{1} M$. Conversely, we will show that if $g=\left(g_{k}\right) \in F_{1}\left(U^{L} ; M\right)$, then each $g_{k}$ is indeed a linear combination of the functions $h_{i j}^{\prime}$.

If $g=\left(g_{k}\right) \in F_{1}\left(U^{L} ; M\right)$, then $U^{L}(f) g=0$ whenever in particular $f \in \operatorname{Ker}_{2} M\left(=L_{2}(G) \cap \operatorname{Ker}_{1} M\right)$. Hence, for $f \in \operatorname{Ker}_{2} M, k=1, \cdots, r$, we have $\int_{G} f(\gamma) g_{k}\left(a \gamma^{-1}\right) d \gamma=\left(f_{\circ} g_{k}\right)(a)=0$ for almost all $a \in G$, and since the convolutions $f \circ g_{k}$ are continuous we have $\left(f \circ g_{k}\right)(\alpha)=0$ for all $a \in G$. Letting $a=e$, we infer that if $U^{L}(f) g=0$ for $f \in \operatorname{Ker}_{2} M$, then $\int_{G} f(\gamma) g_{k}\left(\gamma^{-1}\right) d \gamma=0, k=1, \cdots, r$.

Define, for each $h_{i j}, g_{k} \in L_{2}(G)$, linear functionals $F_{i j}, F_{k}$ on $L_{2}(G)$ as follows:

$$
\begin{array}{rlrl}
F_{i j}(f) & =\int_{G} f(\gamma) h_{i j}(\gamma) d \gamma, & i, j=1, \cdots, m \\
F_{k}(f)=\int_{G} f(\gamma) g_{k}\left(\gamma^{-1}\right) d \gamma, & k=1, \cdots, r .
\end{array}
$$


Hence $\operatorname{Ker} F_{k} \supseteqq \bigcap_{i, j=1}^{m} \operatorname{Ker} F_{i j}, k=1, \cdots, r$. We conclude that there exist constants $c_{k i j}$ such that for $k=1, \cdots, r$, we have

$$
F_{k}=\sum_{i, j=1}^{m} c_{k i j} F_{i j},
$$

whence $g_{k}=\sum_{i, j=1}^{m} c_{k i j} h_{i j}^{\prime}$, as we wanted to show.

For purposes of generalization, we extract the following corollary from the above proof.

Corollary. Let $G, H, M$ and $L$ be as in Lemma 1. For any $u, v$ in $H(M)$ let $g_{u, v}$ be the function on $G$ defined by $g_{u, v}(\gamma)=(M(\gamma) u, v)$ for all $\gamma \in G$. Let $S_{M}$ be the linear span of all such functions $g_{u, v}$. Let $S_{M}^{r}$ be the collection of all functions $g$ mapping $G$ into $H(L)$ (with $r=\operatorname{dim} H(L)$ ) which can be written (relative to some fixed basis of $H(L))$ as column-vector valued functions with entries $g_{1}, \cdots, g_{r}$ in $S_{M}$. Then

$$
\begin{aligned}
F_{1}\left(U^{L} ; M\right) & =F_{1}\left(W^{L} ; M\right)=\left\{g \in S_{M}^{r}: g(x \gamma)\right. \\
& =L(x) g(\gamma) \text { for all } x \in H, \gamma \in G\} .
\end{aligned}
$$

4. The space $F_{0}\left(W^{L} ; M\right) \otimes^{*} H(L)^{c}$. Let $G$ be a locally compact group and let $H=\{e\}$. If $M$ is an irreducible unitary representation of $G$ and if $L$ is the trivial irreducible representation of $H$, one calculates $F_{1}\left(W^{L} ; M\right)$ to be the space

$$
\begin{aligned}
F_{1}\left(W^{L} ; M\right)= & \left\{g \in L_{\infty}(G): \int_{G} f(\gamma) g\left(\gamma^{-1}\right) d \gamma=0 \text { for all } f \in L_{1}(G)\right. \text { such that } \\
& \left.\int_{G} f(\gamma)\left(M\left(\gamma^{-1}\right) u, v\right) d \gamma=0 \text { for all } u, v \in H(M)\right\} .
\end{aligned}
$$

Elementary considerations thus show that $F_{1}\left(W^{L} ; M\right)$ is just the weak-* closure in $L_{\infty}(G)$ (= the dual of $L_{1}(G)$ ) of the space $S_{M}=$ linear $\operatorname{span}\left\{g_{u, v} \in L_{\infty}(G): g_{u, v}(\gamma)=(M(\gamma) u, v), u, v \in H(M)\right\}$. Because the weak-* closure of such a space is a very difficult thing to determine precisely, we feel constrained to consider the simpler space $S_{M}\left(=F_{1}\left(W^{L} ; M\right)\right.$ when $M$ is of finite degree).

Let us now suppose that $H$ is any closed subgroup of $G$ with irreducible unitary representation $L$ of finite degree $r$. We define a space $F_{0}\left(W^{L} ; M\right)$ as follows:

$$
F_{0}\left(W^{L} ; M\right)=\left\{g \in S_{M}^{r}: g(x \gamma)=L(x) g(\gamma) \text { for all } x \in H, \gamma \in G\right\},
$$

where $S_{M}^{r}$ is as in the corollary of Proposition 1. Of course, when $G$ is compact, $F_{1}\left(U^{L} ; M\right)=F_{1}\left(W^{L} ; M\right)=F_{0}\left(W^{L} ; M\right)$.

Suppose $\mathscr{C}_{0}$ is a pre-Hilbert space with inner product (,). 
Define a new pre-Hilbert space $\mathscr{H}_{0}^{c}$ as follows: (1) the underlying space of $\mathscr{H}_{0}^{c}$ is $\mathscr{H}_{0} ;$ (2) if $v \in \mathscr{H}_{0}$, the corresponding member of $\mathscr{H}_{0}^{c}$ is denoted by $\bar{v}$; (3) scalar multiplication is given by $a \bar{v}=\overline{\bar{a} v}$; (4) the inner product is given by $(\bar{u}, \bar{v})=(v, u)$. When $\mathscr{H}_{0}$ is a Hilbert space, $\mathscr{H}_{0}^{c}$ is just the conjugate space of $\mathscr{H}_{0}$. Let $\mathscr{H}_{1}, \mathscr{C}_{2}$ be pre-Hilbert spaces containing the elements $u$, $v$, respectively. Define an operator $u \otimes \bar{v}$ of rank 1 from $\mathscr{K}_{2}$ into $\mathscr{J}_{1}$ by $(u \otimes \bar{v}) w=$ $(w, v) u$ for all $w \in \mathscr{J}_{2}$. The space of linear combinations of such operators is just the space of linear operators of finite rank mapping $\mathscr{H}_{2}$ into $\mathscr{H}_{1}$, and is isomorphic to the algebraic tensor product of $\mathscr{C}_{1}$ and $\mathscr{K}_{2}^{c}$; we denote this space by $\mathscr{H}_{1} \otimes \mathscr{H}_{2}^{c}$.

We thus see that $F_{0}\left(W^{L} ; M\right) \otimes H(L)^{c}$ can be regarded as the space of all matrix-valued functions $N$ on $G$ such that the matrix entries of $N$ are functions in $S_{M}$ and such that $N(x \gamma)=L(x) N(\gamma)$ for all $x \in H, \gamma \in G$. If $e_{1}, \cdots, e_{r}$ is an orthonormal basis of $H(L)$ and if $N \in F_{0}\left(W^{L} ; M\right) \otimes H(L)^{c}$, then the matrix entries of $N,\left(N(\cdot) e_{i}, e_{j}\right)$, $i, j=1, \cdots, r$, have the form

$$
\left(N(\cdot) e_{i}, e_{j}\right)=\sum_{k}\left(M(\cdot) v_{k i j}, u_{k i j}\right), v_{k i j}, u_{k i j} \in H(M) .
$$

Since the collection of all these $v_{k i j}$ is finite, we can write

$$
\left(N(\cdot) e_{i}, e_{j}=\sum_{k=1}^{n}\left(M(\cdot) v_{k}, u_{k i j}^{\prime}\right)\right.
$$

where the $v_{k}$ are linearly independent and independent of the particular indices $i, j$. These considerations lead naturally to a definition.

DeFinition 2. Let $G$ be a locally compact group with closed subgroup $H$. Let $M, L$ be irreducible unitary representations of $G, H$, respectively, where $H(L)$ is separable. Define the space $F_{0}\left(W^{L} ; M\right) \otimes * H(L)^{c}$ to be the space of all functions $N$ on $G$ such that:

(a) For all $\gamma \in G, N(\gamma)$ is a trace-class operator on the Hilbert space $H(L)$.

(b) $N(x \gamma)=L(x) N(\gamma)$ for all $x \in H, \gamma \in G$.

(c) There exists an orthonormal basis (ONB) $\left\{e_{i}\right\}$ of $H(L)$ and a finite collection of linearly independent elements $v_{1}, \cdots, v_{n}$ in $H(M)$ such that

$$
\left(N(\cdot) e_{i}, e_{\jmath}\right)=\sum_{k=1}^{n}\left(M(\cdot) v_{k}, u_{k i j}\right), \quad i, j=1,2, \cdots
$$

It is clear that $F_{0}\left(W^{L} ; M\right) \otimes^{*} H(L)^{c}=F_{1}\left(W^{L} ; M\right) \otimes H(L)^{c}$ when $L$ is of finite degree. 
We will need a few more definitions and results. If $\mathscr{Y}$ is a Hilbert space, let $\mathscr{L}(\mathscr{H})$ be the space of all the bounded linear operators mapping $\mathscr{\mathscr { C }}$ into itself and let $\mathscr{L}_{1}(\mathscr{H})$ be the subspace of $\mathscr{L}(\mathscr{H})$ consisting of all the trace-class operators in $\mathscr{L}(\mathscr{C})$. If $\mathrm{Tr}$ denotes the usual trace functional and if $T \in \mathscr{L}_{1}(\mathscr{\mathscr { C }})$, the functional $A \rightarrow \operatorname{Tr}(T A)$ on $\mathscr{L}(\mathscr{C})$ is continuous in the ultraweak topology of $\mathscr{L}(\mathscr{L})$ (see [2]).

Lemma 2. If $G$ is a group with irreducible unitary representation $R$ and if $T \in \mathscr{L}_{1}(H(R))$ satisfies $\operatorname{Tr}(T R(\gamma))=0$ for all $\gamma \in G$, then $T=0$.

Proof. By the von Neumann density theorem, the space $\mathfrak{U}=$ linear span $\{R(\gamma): \gamma \in G\}$ is ultraweakly dense in $\mathscr{L}(H(R))$. Since $\operatorname{Tr}(T A)=0$ for all $A \in \mathfrak{A}$, the ultraweak continuity of $\operatorname{Tr}(T \cdot)$ implies $\operatorname{Tr}(T A)=0$ for all $A \in \mathscr{L}(H(R))$. Therefore $\operatorname{Tr}\left(T T^{*}\right)=0$, so that $T=0$.

We finally recall the following special form of the principle of $n$-fold transitivity: Let $G$ be a group with irreducible representation $R$ and let $\mathfrak{A}=$ linear $\operatorname{span}\{R(\gamma): \gamma \in G\}$. Let $v_{1}, \cdots, v_{n}$ be a collection of linearly independent elements of $H(R)$ and let $v$ be any other element in $H(R)$. Then there exist operators $A_{k} \in \mathfrak{A}$ such that $A_{k} v_{i}=\delta_{i k} v, i, k=1, \cdots, n$, where $\delta_{i k}$ is the Kronecker delta.

Proposition 2. Let $G$ be a locally compact group with closed subgroup $H$. Let $M, L$ be irreducible unitary representations of $G, H$, respectively, where $H(L)$ is separable. Suppose $N$ is a function mapping $G$ into $\mathscr{L}_{1}(H(L)$ ) such that $N(x \gamma)=L(x) N(\gamma)$ for all $x \in H$, $\gamma \in G$. Then the following two statements are equivalent:

(i) There exists $A \in H(M) \otimes H(M)^{c}$ such that $\operatorname{Tr}(N(\gamma))=$ $\operatorname{Tr}(M(\gamma) A)$ for all $\gamma \in G$.

(ii) $N \in F_{0}\left(W^{L} ; M\right) \otimes^{*} H(L)^{c}$.

Proof. (a) (i) $\Rightarrow$ (ii). Writing $A$ in the form $A=\sum_{i=1}^{n} v_{i} \otimes \bar{u}_{i}$, where $v_{1}, \cdots, v_{n}$ are linearly independent elements in $H(M)$, we have, for all $\gamma \in G$,

$$
\operatorname{Tr}(N(\gamma))=\sum_{i=1}^{n}\left(M(\gamma) v_{i}, u_{i}\right)
$$

Let $v$ be any element of $H(M)$. By the principle of $n$-fold transitivity there exist operators $A_{v, k}, k=1, \cdots, n$, of the form $\sum_{p} a_{p} M\left(\gamma_{p}\right)$ such that $A_{v, k} v_{i}=\delta_{\imath k} v$. For $k=1, \cdots, n, \gamma \in G$, we define operators 
$N(v, k ; \gamma)=\sum_{p} a_{p} N\left(\gamma \gamma_{p}\right)$ satisfying conditions (a), (b) of Definition 2. We have

$$
\operatorname{Tr}(N(v, k ; \gamma))=\sum_{i=1}^{n}\left(M(x \gamma) A_{v, k} v_{i}, u_{i}\right)=\left(M(\gamma) v, u_{k}\right) .
$$

Hence, for all $x \in H$, we see that

$$
\operatorname{Tr}\left(\left(\sum_{i=1}^{n} N\left(v_{i}, i ; \gamma\right)\right) L(x)\right)=\sum_{i=1}^{n}\left(M(x \gamma) v_{i}, u_{i}\right)=\operatorname{Tr}(N(\gamma) L(x)) .
$$

Lemma 2 implies $N(\cdot)=\sum_{\imath=1}^{n} N\left(v_{\imath}, i ; \cdot\right)$.

Let $\left\{e_{i}\right\}$ be an ONB of $H(L)$. We will have shown that $N$ satisfies (c) of Definition 2, if, for each $k=1, \cdots, n$, it is the case that $\left(N\left(v_{k}, k ; \cdot\right) e_{i}, e_{j}\right)=\left(M(\cdot) v_{k}, v_{k i j}\right)$, where $u_{k i j} \in H(M)$. To show this, observe that for all $v \in H(M), x \in H, \gamma \in G$,

$$
\left.\overline{\operatorname{Tr}\left(N(v, k ; \gamma)^{*} L(x)\right.}\right)=\operatorname{Tr}\left(N\left(v, k ; x^{-1} \gamma\right)\right)=\left(M(\gamma) v, M(x) u_{k}\right) .
$$

Hence, for $x_{p} \in H$ and scalars $b_{p}$, we have

$$
\operatorname{Tr}\left(N(v \cdot k ; e)^{*} \sum_{p} b_{p} L\left(x_{p}\right)\right)=\left(\sum_{p} b_{p} M\left(x_{p}\right) u_{k}, v\right) .
$$

By the von Neumann density theorem, the space of operators of the form $\sum_{p} b_{p} L\left(x_{p}\right)$ is ultraweakly dense in $\mathscr{L}(H(L))$. Choose a sequence of operators $A_{n i j}$ (of the form $\sum_{p} b_{n p} L\left(x_{n p}\right)$ ) such that $A_{n i j} \rightarrow E_{i j}$ ultraweakly (where $E_{i j}$ is defined by $E_{i j} e_{m}=\delta_{i m} e_{i}$ )。 Let $w_{n i j}=$ $\sum_{p} b_{n p} M\left(x_{n p}\right) u_{k}$. Since $\operatorname{Tr}\left(N(v, k ; e)^{*} A_{n i j}\right)=\left(w_{n i j}, v\right)$ for all $v \in H(M)$, the ultraweak continuity of the trace functional implies that

$$
\left|\left(w_{n i j}-w_{m i j}, v\right)\right| \rightarrow 0
$$

as $n, m \rightarrow \infty$; i.e., $\left\{w_{n i j}\right\}$ is a weak Cauchy sequence. Since $H(M)$ is reflexive and thus weakly complete, there exists $u_{k i j} \in H(M)$ such that

$$
\begin{aligned}
\left(u_{k i j}, M(\gamma) v\right) & =\lim _{n \rightarrow \infty}\left(w_{n i j}, M(\gamma) v\right)=\lim _{n \rightarrow \infty} \operatorname{Tr}\left(N(v, k ; \gamma)^{*} A_{n i j}\right) \\
& \left.=\operatorname{Tr}\left(N(v, k ; \gamma)^{*} E_{i j}\right)=N(v, k ; \gamma)^{*} e_{j}, e_{i}\right) .
\end{aligned}
$$

This is,

$$
\left(N\left(v_{k}, k ; \gamma\right) e_{i}, e_{j}\right)=\left(M(\gamma) v_{k}, u_{k i j}\right) .
$$

(b) (ii) $\Rightarrow$ (i). If $N \in F_{0}\left(W^{L} ; M\right) \otimes^{*} H(L)^{c}$, there exist linearly independent elements $v_{1}, \cdots, v_{n}$ in $H(M)$, an ONB $\left\{e_{i}\right\}$ of $H(L)$, and elements $u_{k i j} \in H(M)$ such that

$$
\left(N(\gamma) e_{i}, e_{j}\right)=\sum_{k=1}^{n}\left(M(\gamma) v_{k}, u_{k i j}\right), \quad i, j=1,2, \cdots
$$


Exactly as in (a), use $n$-fold transitivity to define, for all $v \in H(M)$, $k=1, \cdots, n$, functions $N(v, k ; \cdot) \in F_{0}\left(W^{L} ; M\right) \otimes * H(L)^{c}$ satisfying

$$
\left(N(v, k ; \cdot) e_{i}, e_{j}\right)=\left(M(\cdot) v, u_{k i j}\right), \quad i, j=1,2, \cdots .
$$

Let $E_{m}$ be the projection on the space spanned by $e_{1}, \cdots, e_{m}$; since $H(L)$ is separable, these $E_{m}$ converge ultraweakly to the identity operator on $H(L)$. We see that

$$
\operatorname{Tr}\left(N(v, k ; e) E_{m}\right)=\sum_{i=1}^{m}\left(v, u_{k i i}\right)=\left(v, u_{k m}\right),
$$

where the $u_{k m}=\sum_{i=1}^{m} u_{k i i}$ must converge weakly to an element $u_{k}$ in $H(M)$ and $\operatorname{Tr}(N(v, k ; e))=\left(v, u_{k}\right)$. For $v=M(\gamma) v_{k}$ (so that $N(v, k ; e)=$ $\left.N\left(v_{k}, k ; \gamma\right)\right)$ we see that

$$
\operatorname{Tr}\left(N\left(v_{k}, k ; \gamma\right)\right)=\left(M(\gamma) v_{k}, u_{k}\right)=\operatorname{Tr}\left(M(\gamma)\left(v_{k} \otimes \bar{u}_{k}\right)\right) .
$$

Letting $A=\sum_{k=1}^{n} v_{k} \otimes \bar{u}_{k} \in H(M) \otimes H(M)^{c}$, we have, for all $\gamma \in G$, $\operatorname{Tr}(N(\gamma))=\operatorname{Tr}\left(\sum_{k=1}^{b} N\left(v_{k}, k ; \gamma\right)\right)=\operatorname{Tr}(M(\gamma) A)$.

5. A quasi-null space. Let $G$ be a locally compact group with unitary representations $R_{1}$ and $R_{2}$, where $R_{2}$ is irreducible. We will define a subspace $Q F\left(R_{1} ; R_{2}\right)$ of $H\left(R_{1}\right)$, called the quasi-null space coming from $R_{1}$ at $R_{2}$, as follows: $Q F\left(R_{1} ; R_{2}\right)=\left\{u \in H\left(R_{1}\right)\right.$ : for all $\gamma \in G$ and all $v \in H\left(R_{1}\right)$, there exists an operator $T(u, v) \in \mathscr{E}_{1}\left(H\left(R_{2}\right)\right)$ such that $\left.\left(R_{1}(\gamma) u, v\right)=\operatorname{Tr}\left(T(u, v)^{*} R_{2}(\gamma)\right)\right\}$. If $u \in Q F\left(R_{1} ; R_{2}\right)$, we have for all $v \in H\left(R_{1}\right)$ and all $f \in L(G)$ that

$$
\begin{aligned}
\left(R_{1}(f) u, v\right) & =\int_{G} f(\gamma)\left(R_{1}\left(\gamma^{-1}\right) u, v\right) d \gamma \\
& =\operatorname{Tr}\left(T(u, v)^{*} R_{2}(f)\right),
\end{aligned}
$$

so that $\left(R_{1}(f) u, v\right)=\operatorname{Tr}\left(T(u, v)^{*} R_{2}(f)\right)$ for all $f \in C^{*}(G)$. Thus $f \in \operatorname{Ker} R_{2}$ implies $R_{1}(f) u=0$. We see that $Q F\left(R_{1} ; R_{2}\right) \subseteq F\left(R_{1} ; R_{2}\right)$.

Proposition 3. Let $G$ be a locally compact group. Let $R_{1}$ be a unitary representation of $G$ and let $R_{2}$ be an irreducible unitary representation of $G$ such that $R_{2}(f)$ is a compact operator for all $f \in C^{*}(G)$. Then $Q F\left(R_{1} ; R_{2}\right)=F\left(R_{1} ; R_{2}\right)$.

Proof. Let $u \in F\left(R_{1} ; R_{2}\right)$. We have $\left(R_{1}(f) u, v\right)=0$ for all $v \in H\left(R_{1}\right)$ and for all $f \in \operatorname{Ker} R_{2}$. For any $v \in H\left(R_{2}\right)$, the continuous functional $F_{u, v}$ on $C^{*}(G)$ defined by $F_{u, v}(f)=\left(R_{1}(f) u, v\right)$ therefore induces a continuous functional $F_{u, v}^{\prime}$ on the quotient space $C^{*}(G) / \operatorname{Ker} R_{2}$. As $R_{2}$ is compact, the mapping $f \rightarrow R_{2}(f)$ maps $C^{*}(G)$ onto $\mathscr{L}_{c}\left(H\left(R_{2}\right)\right)$ 
(= the space of compact operators on $H\left(R_{2}\right)$ ), and thus induces an isomorphism

$$
R_{2}^{\sharp}: C^{*}(G) / \operatorname{Ker} R_{2} \cong \mathscr{L}_{c}\left(H\left(R_{2}\right)\right) \text {. }
$$

Since $\mathscr{L}_{c}\left(H\left(R_{2}\right)\right)$ is a $C^{*}$-algebra this isomorphism is an isometry ([3, pp. 18, 82]). For $f \in C^{*}(G)$, let $f^{*}$ denote its equivalence class in $C^{*}(G) / \operatorname{Ker} R_{2}$. Using the well-known fact that the dual of $\mathscr{L}_{c}\left(H\left(R_{2}\right)\right)$ is $\mathscr{L}_{1}\left(H\left(R_{2}\right)\right)([3$, p. 82]), we conclude that there exists a trace-class operator $T(u, v)^{*}$ such that $F_{u, v}^{\prime}\left(f^{\sharp}\right)=\operatorname{Tr}\left(T(u, v)^{*} R_{2}^{\sharp}\left(f^{\sharp}\right)\right)$ for all $f^{\sharp} \in$ $C^{*}(G) / \operatorname{Ker} R_{2}$; i.e., $\left(R_{1}(f) u, v\right)=\operatorname{Tr}\left(T(u, v)^{*} R_{2}(f)\right)$ for all $f \in C^{*}(G)$. A simple convergence argument shows that $\left(R_{1}(\gamma) u, v\right)=\operatorname{Tr}\left(T(u, v)^{*} R_{2}(\gamma)\right)$ for all $\gamma \in G$; i.e., $u \in Q F\left(R_{1} ; R_{2}\right)$.

REMARK. When $R_{2}$ above is of finite degree, one shows by a similar technique that $Q F\left(R_{1} ; R_{2}\right)=F_{1}\left(R_{1} ; R_{2}\right)$.

6. A Frobenius reciprocity theorem. Let $G$ be a locally compact group with closed subgroup $H$. Let $M, L$ be irreducible unitary representations of $G, H$, respectively. For $A \in H(M) \otimes H(M)^{c}$, write $A=\sum_{i=1}^{n} v_{i} \otimes \bar{u}_{i}$, where $v_{1}, \cdots, v_{n}$ are linearly independent in $H(M)$. Let us observe the equivalence of the following two statements:

(i) For each $\gamma \in G$, there exists $T_{A}(\gamma) \in \mathscr{L}_{1}(H(L))$ such that $\operatorname{Tr}(M(x \gamma) A)=\operatorname{Tr}\left(T_{A}(\gamma) L(x)\right)$ for all $x \in H$.

(ii) $\quad A \in H(M) \otimes Q F\left(M_{H} \text {; } L\right)^{c}$.

To show (i) $\Rightarrow$ (ii), note that for all $x \in H, \gamma \in G$,

$$
\operatorname{Tr}\left(M\left(x^{-1} \gamma\right) A\right)=\sum_{i=1}^{n}\left(M\left(x^{-1} \gamma\right) v_{i}, u_{i}\right)=\operatorname{Tr}\left(T_{A}(\gamma) L\left(x^{-1}\right)\right)
$$

As in the proof of Proposition 2, use $n$-fold transitivity to find, for all $v \in H(M), k=1, \cdots, n$, operators of the form $\sum_{p} a_{p} M\left(\gamma_{p}\right)$, for which

$$
\left(M(x) u_{k}, v\right)=\operatorname{Tr}\left(T\left(u_{k}, v\right) * L(x),\right.
$$

where

$$
T\left(u_{k}, v\right)=\sum_{p} a_{p} T_{A}\left(\gamma_{p}\right) \in \mathscr{L}_{1}(H(L)) .
$$

Conversely, for $A=\sum_{i=1}^{n} v_{i} \otimes \bar{u}_{i}$ with $u_{1}, \cdots, u_{n} \in Q F\left(M_{H} ; L\right)$, if we let $T_{A}(\cdot)=\sum_{i=1}^{n} T\left(u_{i}, M(\cdot) v_{i}\right)$ statement $\mathrm{i}$ follows.

It is easy to see that $T_{A}(\cdot)$ in $\mathrm{i}$ is unique. Indeed, if $T_{A}^{\prime}(\cdot)$ also satisfies $\mathrm{i}$, the fact that $\operatorname{Tr}\left(\left(T_{A}(\gamma)-T_{A}^{\prime}(\gamma)\right) L(x)\right)=0$ for all $x \in H$, $\gamma \in G$, implies, by Lemma 2 , that $T_{A}^{\prime}=T_{A}$. It follows that the map $A \rightarrow T_{A}$ is linear. 
THEOREM 2. Let $G$ be a locally compact group with closed subgroup $H$. Let $M$ be an irreducible unitary representation of $G$ and $L$ an irreducible unitary representation of $H$, where $H(L)$ is separable. Then the linear map $A \rightarrow T_{A}$ maps $H(M) \otimes Q F\left(M_{H} ; L\right)^{c}$ isomorphically onto $F_{0}\left(W^{L} ; M\right) \otimes^{*} H(L)^{c}$.

Proof. The map $A \rightarrow T_{A}(\cdot)$ is injective, since $T_{A}(\cdot)=0$ implies $\operatorname{Tr}(M(\gamma) A)=0$ for all $\gamma \in G$; by Lemma $2, A=0$. Let $A \in H(M) \otimes$ $Q F\left(M_{H} ; L\right)^{c}$. For all $x, y \in H, \quad \gamma \in G$, we have $\operatorname{Tr}\left(T_{A}(x \gamma) L(y)\right)=$ $\operatorname{Tr}(M(y) M(x \gamma) A)=\operatorname{Tr}\left(L(x) T_{A}(\gamma) L(y)\right)$. By Lemma 2, $T_{A}(x \gamma)=L(x) T_{A}(\gamma)$. By Proposition 2, the fact that $\operatorname{Tr}\left(T_{A}(\gamma)\right)=\operatorname{Tr}(M(\gamma) A)$ for all $\gamma \in G$ implies that $T_{A} \in F_{0}\left(W^{L} ; M\right) \otimes * H(L)^{c}$.

To see that $A \rightarrow T_{A}$ is surjective, let $N \in F_{0}\left(W^{L} ; M\right) \otimes^{*} H(L)^{c}$. By Proposition 2, there exists $A \in H(M) \otimes H(M)^{c}$ such that for all $x \in H, \quad \gamma \in G, \quad \operatorname{Tr}(N(\gamma) L(x))=\operatorname{Tr}(N(x \gamma))=\operatorname{Tr}(M(x \gamma) A)$, so that by the equivalence of statements (i) and (ii) preceding this theorem, we have $A \in H(M) \otimes Q F\left(M_{H} ; L\right)^{c}$. By the uniqueness of $T_{A}$ we must have $N=T_{A}$, as we needed to show.

We have already noted that when $L$ and $M$ are of finite degree, we have $F_{0}\left(W^{L} ; M\right)=F_{1}\left(W^{L} ; M\right)$ and $Q F\left(M_{H} ; L\right)=F\left(M_{H} ; L\right)=F_{1}\left(M_{H} ; L\right)$. Indeed, if $L$ is compact, $Q F\left(M_{H} ; L\right)=F\left(M_{H} ; L\right)$. Further, if $G$ is compact, $F_{1}\left(W^{L} ; M\right)=F_{1}\left(U^{L} ; M\right)=F\left(U^{L} ; M\right)$. In light of Theorem 1 , we have a complete generalization as well as another proof of the classical Frobenius Reciprocity Theorem.

It is to be hoped that this approach somehow lays bare the skeleton of the structure of this reciprocity situation, and, moreover, that the quasinull space yields some information as the manner in which an irreducible representation is contained in some other representation.

The author wishes to express his gratitude for the help and guidance given him by R. J. Blattner, under whom this work was developed as a part of the author's doctoral dissertation at the University of California, Los Angeles.

\section{REFERENCES}

1. R. J. Blattner, Group extension representations and the structure space, Pacific J. Math. 15 (1965), 1101-1113.

2. J. Dixmier, Les algèbres d'opérateurs dans l'espace hilbertien, Gauthier-Villars, Paris, 1957.

3. - Les $C^{*}$-algèbres et leurs reprèsentations, Gauthier-Villars, Paris, 1964.

4. J. M. G. Fell, The dual spaces of $C^{*}$-algebras, Trans. Amer. Math. Soc. 94 (1960), 365-403. 
5. J. Glimm, Families of induced representations, Pacific J. Math. 12 (1962), 885-911. 6. - Type I C*-algebras, Ann. of Math. 73 (1961), 572-612.

7. A. Kleppner, Intertwining forms for summable induced representations, Trans. Amer. Math. Soc. 112 (1964), 164-183.

8. C. C. Moore, On the Frobenius reciprocity theorem for locally compact groups, Pacific J. Math. 12 (1962), 359-365.

Received October 10, 1969.

University of California, Los ANgeles, AND

California State College, Dominguez Hills 



\title{
PACIFIC JOURNAL OF MATHEMATICS
}

\author{
EDITORS
}

H. SAMELSON

Stanford University

Stanford, California 94305

C. R. Новву

University of Washington

Seattle, Washington 98105
J. DUGUNDJI

Department of Mathematics

University of Southern California

Los Angeles, California 90007

RICHARD ARENS

University of California

Los Angeles, California 90024

\section{ASSOCIATE EDITORS}

E. F. BECKENBACH

B. H. NeUManN

F. WOLF

K. YosHidA

\section{SUPPORTING INSTITUTIONS}

UNIVERSITY OF BRITISH COLUMBIA CALIFORNIA INSTITUTE OF TECHNOLOGY UNIVERSITY OF CALIFORNIA MONTANA STATE UNIVERSITY UNIVERSITY OF NEVADA NEW MEXICO STATE UNIVERSITY OREGON STATE UNIVERSITY UNIVERSITY OF OREGON OSAKA UNIVERSITY UNIVERSITY OF SOUTHERN CALIFORNIA
STANFORD UNIVERSITY UNIVERSITY OF TOKYO UNIVERSITY OF UTAH WASHINGTON STATE UNIVERSITY UNIVERSITY OF WASHINGTON

AMERICAN MATHEMATICAL SOCIETY CHEVRON RESEARCH CORPORATION TRW SYSTEMS

NAVAL WEAPONS CENTER 


\section{Pacific Journal of Mathematics}

\section{Vol. 36, No. $1 \quad$ November, 1971}

Norman Larrabee Alling, Analytic and harmonic obstruction on

nonorientable Klein surfaces ............................ 1

Shimshon A. Amitsur, Embeddings in matrix rings .............. 21

William Louis Armacost, The Frobenius reciprocity theorem and essentially bounded induced representations ....................... 31

Kenneth Paul Baclawski and Kenneth Kapp, Topisms and induced

non-associative systems ............................ 45

George M. Bergman, The index of a group in a semigroup ............ 55

Simeon M. Berman, Excursions above high levels for stationary Gaussian

processes....................................... 63

Peter Southcott Bullen, A criterion for $n$-convexity .............. 81

W. Homer Carlisle, III, Residual finiteness of finitely generated commutative

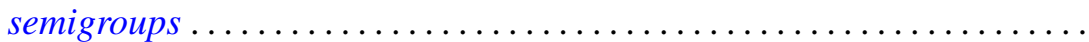

Roger Clement Crocker, On the sum of a prime and of two powers of

two ............................................ 103

David Eisenbud and Phillip Alan Griffith, The structure of serial rings . . . 109

Timothy V. Fossum, Characters and orthogonality in Frobenius

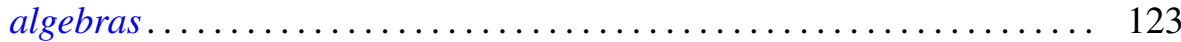

Hugh Gordon, Rings of functions determined by zero-sets . .......... 133

William Ray Hare, Jr. and John Willis Kenelly, Characterizations of Radon partitions...

Philip Hartman, On third order, nonlinear, singular boundary value

problems

David Michael Henry, Conditions for countable bases in spaces of

countable and point-countable type ..

James R. Holub, Hilbertian operators and reflexive tensor products ...

Robert P. Kaufman, Lacunary series and probability ..... . .

195

Erwin Kreyszig, On Bergman operators for partial differential equations in

two variables ................................

Chin-pi Lu, Local rings with noetherian filtrations . .

Louis Edward Narens, A nonstandard proof of the Jordan curve theorem...

S. P. Philipp, Victor Lenard Shapiro and William Hall Sills, The Abel summability of conjugate multiple Fourier-Stieltjes integrals. .

Joseph Earl Valentine and Stanley G. Wayment, Wilson angles in linear normed spaces

Hoyt D. Warner, Finite primes in simple algebras ...

Horst Günter Zimmer, An elementary proof of the Riemann hypothesis for an elliptic curve over a finite field... 